\title{
A Reconstructed Phase Space Approach for Distinguishing Ischemic from Non- Ischemic ST Changes using Holter ECG Data
}

\author{
MW Zimmerman, RJ Povinelli, MT Johnson, KM Ropella \\ Marquette University, Milwaukee, WI, USA
}

\begin{abstract}
A method for the classification of ST events through the use of reconstructed phase spaces of the ECG signal is proposed. There is a clinical need for the creation of an automated system for classification of ST events as ischemic or non-ischemic as existing ischemia detection methods are expensive, invasive, or both. The algorithm proposed herein attempts to classify events using the 16 beats surrounding a given ST event. The ST segment and $T$ wave of each of these beats is embedded in a phase space and then modelled and classified through the use of Gaussian Mixture Models (GMM). Using ten-fold cross validation of available training data the sensitivity and specificity were $81.0 \%$ and $88.1 \%$ respectively.
\end{abstract}

\section{Introduction}

Myocardial ischemia is a common heart condition that is often difficult to detect from routine ECG recordings. Ischemia occurs when oxygen and nutrient-rich blood is not adequately supplied to part of the cardiac tissue. This deficiency takes only a short time to lead to infarction (tissue death). Myocardial infarction and ischemia can lead to serious complications such as lethal arrhythmias and heart failure [1]. Early detection of ischemia is crucial to preserving cardiac tissue and improving patient health.

There are several methods currently employed for the detection of myocardial ischemia. The most accurate method for detection is coronary angiography. This, the most invasive method of detection, requires the insertion of a catheter into the blood vessels of the heart. Another common method for detection is called exercise testing. This method requires the patient to perform rigorous exercise in order to increase heart rate and blood flow [2]. The heart is examined using an echocardiogram to look for abnormalities in heart function. This method requires at least three personnel and has accuracies of detection around $85 \%$ [3]. Due to the cost and difficulty in performing angiographies and echocardiograms, patients are examined for myocardial ischemia only if they are experiencing chest pains or thought to be at high risk.
This can lead to missed diagnosis, as ischemia often causes no pain to the patient.

Electrocardiogram (ECG) recordings are a noninvasive method for monitoring the electrical signals of a patient's heart. The ECG can provide information for diagnosing cardiac abnormalities. The correlation between changes in the ECG signal and ischemia often been explored.

The loss of life-sustaining oxygen to cardiac tissue causes changes in the depolarization of the heart's ventricular tissue [4]. Because the oxygen-deprived tissue conducts the depolarizing electrical signal abnormally, the period following depolarization can be disrupted. This normally isoelectric period is represented in the ECG waveform as the ST segment (the time between the QRS and repolarizing $\mathrm{T}$ wave). Cardiac tissue that is ischemic or injured does not properly depolarize. This leads to socalled "injury current." This current causes a depression in the T-Q segment and an elevation in the ST segment, which appears on most medical monitoring equipment as a large ST elevation because of processes such as baseline correction [5-7].

Several techniques for detecting myocardial ischemia using ECG signals have been examined. Currently, medical equipment can alert a physician to possible changes in ST and T wave deviations, but an expert must still examine these changes to determine whether they are due to ischemia. Current methods of automatic ischemia classification are very slow and cannot detect ischemia in real-time. This demonstrates a need for an effective and efficient algorithm for automatic ischemia detection [8].

The ischemia classification algorithm presented here was developed in response to the 2003 Computers in Cardiology Challenge. This challenge was to develop an algorithm to determine the difference between transient ST changes that are caused by ischemia and those that are not. Current methods, which use only the ECG signal for ischemia detection, are highly sensitive but have low specificity. For this reason it is desirable to create a method that can provide both high sensitivity and specificity. The data supplied for use in the challenge is the Long-Term ST Database (LTSTDB) and a set of files which define the beginning of ST events to be classified and the channel on which the event was observed.

This paper describes an automated method for 
distinguishing ST events as ischemic or non-ischemic using reconstructed phase spaces of Holter ECG data. A reconstructed phase space (RPS) is a way of extracting the multidimensional data that is embedded in a timeseries signal by plotting the signal against delayed versions of itself [9]. RPS based methods have provided significant results in other fields such as speech recognition. By embedding multiple, consecutive ST segments of ECG and then modeling them with a Gaussian Mixture Model (GMM), an algorithm can make a determination as to whether a given set of heart beats represent ischemic or non-ischemic ST events $[10,11]$.

\section{Challenge}

The goal of the 2003 Computers in Cardiology Challenge was to develop an algorithm that could ascertain whether an ST segment event was produced by ischemic or non-ischemic causes. For this challenge a significant ST episode was defined as follows: "An episode begins when the magnitude of the ST deviation first exceeds $50 \mu \mathrm{V}$. The deviation must reach $100 \mu \mathrm{V}$ or more throughout a continuous interval of at least 30 seconds. The episode ends when the deviation becomes smaller than $50 \mu \mathrm{V}$, provided that it does not exceed $50 \mu \mathrm{V}$ in the following 30 seconds [12]." The significant episode is labeled as: ischemic, axis shift (AS) related, conduction change (CC) related, or heart rate (HR) related ST change. The axis shift, conduction change, and heart rate related episodes are all considered non-ischemic events for this challenge. The start times for each significant episode to be classified have been provided along with the channel number of the affected signal [4].

The performance measure for the challenge was the overall accuracy of the algorithm. One point is given for a correct classification, i.e., non-ischemic event classified as non-ischemic, 1 point is subtracted for an incorrect classification i.e., ischemic event classified as nonischemic, and no points are added or subtracted for an event that is left unclassified or classified as unknown [4].

\section{Long-Term ST Database}

The Long-Term ST Database consists of 86 two or three channel, 21 to 24 hour Holter ECG recordings. These recordings are separated into two groups for this challenge. The first group is a training set of 43 records from 42 patients, which has been available since the start of the challenge. The second group is a test set of 43 more recordings against which the developed algorithm will be given to determine the challenge score. This set of data will be released following the challenge completion [4, 13].

Complete annotations have been provided for the database, which label the significant ST shifts and episodes. The beginning (J-point) of most ST segments has been annotated along with $\mathrm{R}$ wave annotations using a 16 second averaging window. These annotations have been generated using only the first channel of each patient's ECG recording. In cases where the first lead is too noisy to allow for detection, a second detection was made using the WQRS application available in the WFDB applications package from PhysioNet [14]. The WQRS program was applied to the signal on which the challenge specified that the episode was seen.

\section{Reconstructed Phase Space Theory}

The reconstructed phase space (RPS) is used in this algorithm as a way of capturing the embedded trajectory of the signal. Takens [15] has shown that if the dimension of the reconstructed phase space is chosen large enough, a topological equivalent to the original system is created. To create a reconstructed phase space the original signal is plotted against delayed versions of itself. In this procedure the RPS is formed according to the equation

$$
\mathbf{x}_{n}=\left[\begin{array}{llll}
x_{n} & x_{n-\tau} & \cdots & x_{n-(d-1) \tau}
\end{array}\right] \quad n=(1+(d-1) \tau \ldots N
$$

where $\mathbf{x}_{\mathrm{n}}$ is the $\mathrm{n}$ dimensional phase space, $x_{n}$ is the $\mathrm{n}^{\text {th }}$ point in the time series, $\tau$ is the time lag, $N$ is the number of points in the time series, and $d$ is the dimension of the phase space.

\section{Algorithm Design}

The proposed algorithm extracts data from the LongTerm ST Database by first extracting the J-Points. This is first done using the provided 16 beat averaged annotation files. Eight J-points before and eight after the time of the event are collected for 16 total J-Point times. The beats can be described from the indices -8 to 7 . If these times are found to be more than 400 samples (1.6s) from each other, they are determined to be invalid. If any of the Jpoints are found to be invalid, the WQRS program is used. This program, from the WFDB Software Package, uses the length transform to locate the QRS complexes and J-points within a signal [14]. Once the J-points are collected, the 100 samples $(400 \mathrm{~ms})$ of waveform following the J-point are extracted. This time duration was chosen because it captures the ST segment and the T wave for the range of heartbeats found in the database.

After data extraction, each waveform is embedded in a reconstructed phase space. An embedding is done for each index and channel combination as shown in Figure 1. The embedding is done using a dimension of 6 and a time lag of 5 . These parameters were chosen empirically. 


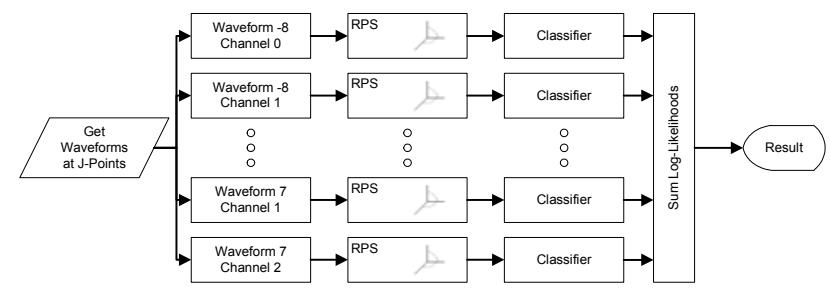

Figure 1 - Proposed Classification System

Prior to any classification, a set of models must be created. This is considered the training portion of this algorithm. For training, the embeddings of matching index, channel, and event type (ischemic, cc or as nonischemic, hr non-ischemic) are combined. A Gaussian Mixture Model (GMM) is learned for each combination of embeddings. For this system, 25 mixtures were used to model the data. The choice of this number was based on estimation of data complexity and experimentation. This process is done using the available training data.

Once the models have been developed on the training data, a classification scheme of the test data is derived. The data is extracted from the database in the manner described above. For a given event time the embedded phase space for each combination of index and channel is classified with a Bayes' classifier that calculates the likelihood of each class. The logs of the likelihoods are then summed across each index and channel. The event is classified as the class with the highest summed loglikelihood as:

$$
\hat{\omega}=\max _{i=1 \ldots C}\left\{\hat{l}_{i}(x)\right\},
$$

where $x$ is the test data vector and $C$ is the number of classes [16].

\section{Results}

\subsection{Learning Data Results}

In order to make full use of all of the training data provided for the challenge the records were split into a development set and a validation set. The validation set consisted of the records: s20221, s20501, s30661, s30741, and s30742. The development set consisted of the remaining 38 records. The development set of the remaining 38 records was partitioned into 10 patient independent folds. Each fold was balance, as best possible, across classes.

\begin{tabular}{lcccc}
\hline \multicolumn{5}{c}{ Classified As } \\
& Ischemic & AS/CC & HR & Sensitivity \\
\hline Ischemic & 268 & 46 & 17 & $81.0 \%$ \\
AS/CC & 111 & 694 & 19 & $84.2 \%$ \\
HR & 3 & 15 & 119 & $86.9 \%$ \\
\hline
\end{tabular}

Table 1 - Confusion Matrix for Development Set
For this challenge, it was important to see how well the classifier could classify between ischemic and nonischemic data. For this reason, the results for the two nonischemic classes (AS/CC and HR) are combined. This helps to increases the sensitivity of the algorithm.

\begin{tabular}{ccc}
\hline Sensitivity & Specificity & Accuracy \\
\hline $81.0 \%$ & $88.1 \%$ & $86.3 \%$ \\
\hline
\end{tabular}

Table 2 - Folded Results for Development Set Data

Upon testing the algorithm on the test portion of the training data, the results were as follows.

\begin{tabular}{lcccc}
\hline \multicolumn{5}{c}{ Classified As } \\
& Ischemic & AS/CC & HR & Sensitivity \\
\hline Ischemic & 58 & 14 & 0 & $80.6 \%$ \\
AS/CC & 85 & 197 & 123 & $48.6 \%$ \\
HR & 1 & 1 & 1 & $33.3 \%$ \\
\hline
\end{tabular}

Table 3 - Confusion Matrix for Validation Set

By combining the results for the two non-ischemic classes the actual results shown below are found.

\begin{tabular}{ccc}
\hline Sensitivity & Specificity & Accuracy \\
\hline $80.6 \%$ & $78.9 \%$ & $79.1 \%$ \\
\hline
\end{tabular}

Table 4 - Folded Results for Validation Set

\subsection{Test Data Results}

The results of the described algorithm being applied to the Computers in Cardiology 2003 Challenge Test data are as follows.

\begin{tabular}{ccc}
\hline Sensitivity & Specificity & Accuracy \\
\hline $63.8 \%$ & $49.9 \%$ & $55.7 \%$ \\
\hline
\end{tabular}

Table 5 - Results for Learning Group Data

\section{Discussion}

There are two hypotheses for the 30.6\% drop in accuracy for the test set data. The first hypothesis is that the code written contains errors. The original development for this project was done in the Matlab environment, while the project code submitted was required to be written in ANSI C. Due to time constraints, it was not possible to completely debug the $\mathrm{C}$ code and some problems were discovered. After the project due date, a difference in J-Point detection and a difference in sample extraction were the two areas where problems were located. Differences discovered after the final code submission date attribute to over a six percent drop in overall accuracy when the $\mathrm{C}$ code is tested on the 
ten-fold cross validation of the training set. It is possible that this problem was amplified due to characteristics of the test set data.

The second hypothesis is that this method captures something in the training data that is not present in the test data. It is difficult to analyze if this is the case since the test data is not yet available. The experiments on the development set of data tend to discredit the theory of a faulty method. During development, the models were never trained on data that was used for the testing. This should have created conditions identical to classification of the test set data. Since all sets of data were patient independent, it is not clear how a faulty algorithm could have received accuracies over 30\% higher than another very similar set of data.

In conclusion, a novel method for classification of ST events as ischemic or non-ischemic has been presented. While there is disagreement in some of the results, the accuracy of the ten fold cross validation is similar to other methods of classification, such as echocardiograms. Once the test set data of the database has been released, the reason for this discrepancy can be analyzed and resolved. This method has shown that it can be effective and that it still needs some work. It is clear from the results that the use of reconstructed phase spaces for ST event classification

\section{References}

[1] Wagner GS. Marriott's practical electrocardiography. Baltimore, MD: Williams \& Wilkins, 1994.

[2] Ischemia: The Thomson Corporation, 2002.

[3] Warnick. WC, John J Ross J, Karalis DG.

Echocardiography: Stress echocardiography in clinical practice. Vol. 2003. Philadelphia, PA: MCP

Hahnemann University, 1996.

[4] Computers in cardiology challenges. Vol. 2003. Cambridge, MA, 2003.

[5] Braunwald E, Maroko P. St-segment mapping. Realistic and unrealistic expectations. Circulation 1976;54:529-532.

[6] Fozzard H, DasGupta D. St-segment potentials and mapping. Theory and experiments. Circulation 1976;54:533-537.

[7] Janse M, Kleber A. Electrophysiological changes and ventricular arrhythmias in the early phase of regional myocardial ischemia. Circulation Research 1981;49:1069-1081.

[8] Papaloukas C, Fotiadis DI, Likas A, Michalis LK. Automated methods for ischemia detection in longduration ecgs. Cardiovascular Reviews and Reports 2003;24:313-320.
[9] Lindgren AC, Johnson MT, Povinelli RJ. Speech recognition using reconstructed phase space features, International Conference on Acoustics, Speech and Signal Processing, Hong Kong, 2003;I:61-63.

[10] Ye J, Povinelli RJ, Johnson MT. Phoneme classification using naive bayes classifier in reconstructed phase space, IEEE Signal Processing Society 10th Digital Signal Processing Workshop, 2002:2.2.

[11] Povinelli RJ, Feng X. A new temporal pattern identification method for characterization and prediction of complex time series events. IEEE Transactions on Knowledge \& Data Engineering 2003;15:339-352.

[12] Jager F, Moody G, Taddei A, et al. Development of a long term database for assessing the performance of transient ischemia detectors. Computers in Cardiology 1996;23:481-484.

[13] Jager F, Taddei A, Moody G, et al. Long-term st database: A reference for the development and evaluation of automated ischaemia detectors and for the study of the dynamics of myocardial ischaemia. Medical and Biological Engineering and Computing 2003;41:172-182.

[14] Moody GB. Wfdb applications guide. Vol. 2003. Cambridge, MA, 2003.

[15] Takens F. Detecting strange attractors in turbulence. In: Mathematics Institute, University of Warwick, Dynamical Systems and Turbulence, Warwick, 1980;898:366-381.

[16] Indrebo KM, Povinelli RJ, Johnson MT. A combined sub-band and reconstructed phase space approach to phoneme classification, ISCA Tutorial and Research Workshop on Non-linear Speech Processing (NOLISP), Le Croisic, France, 2003:107-110.

Address for correspondence.

Michael W. Zimmerman

Olin Engineering, Room 523

1515 W. Wisconsin Ave.

Milwaukee, WI 53233

michael.w.zimmerman@marquette.edu

Richard J. Povinelli

Haggerty Engineering, Room 224

1515 W. Wisconsin Ave.

Milwaukee, WI 53233

richard.povinelli@marquette.edu 